\title{
Numerical Quadrature Over a Rectangular Domain in Two or More Dimensions
}

\author{
Part 3. Quadrature of a Harmonic Integrand
}

\section{By J. C. P. Miller}

1. Introduction. In Part 1 [1], §5, formula (B), §7, formula $\left(\mathrm{B}^{\prime}\right)$, and in $\$ 9$; also in Part 2 [2] in several places, we have seen how the error term is very much reduced if the integrand $f(x, y)$ is a harmonic function, that is, if $\nabla^{2} f=0$. In this note we pursue further this special case, in which especially high accuracy is attainable with few points.

It may not be often that the integrand will have this special form, but it seems worthwhile to develop a few of the interesting formulas. We start by obtaining expansions for $n$ variables, and more extensive ones for two variables, and then obtain and consider special quadrature formulas.

2. Expansions. As in Part 2 [2] §2, we develop $f\left(x_{1}, x_{2}, \cdots, x_{n}\right)$ as a Taylor series in even powers of each of the variables $x_{r}$. Then, using $\nabla^{2} f_{0}=0$ whenever it is applicable, we obtain

$$
\left\{\begin{aligned}
J=I /(2 h)^{n}= & (2 h)^{-n}\left(\int_{-h}^{h}\right)^{n} f\left(x_{1}, x_{2}, \cdots, x_{n}\right) d x_{1} d x_{2} \cdots d x_{n} \\
= & f_{0}+\frac{h^{4}}{5 !} \frac{4}{3} D^{4} f_{0}+\frac{h^{6}}{7 !} \frac{16}{3} \Im^{6} f_{0}+\frac{h^{8}}{9 !}\left(\frac{16}{5} D^{8}+\frac{192}{5} Q^{8}\right) f_{0} \\
& +\frac{h^{10}}{11 !}\left(\frac{128}{3} D^{4} J^{6}+\frac{1280}{3} \mathcal{P}^{10}\right) f_{0} \\
& +\frac{h^{12}}{13 !}\left(\frac{64}{7} D^{12}+\frac{4096}{35} \Im^{12}+\frac{61184}{105} D^{4} Q^{8}+\frac{707584}{105} \mathcal{S}^{12}\right) f_{0} \\
& +\cdots
\end{aligned}\right.
$$

where, as before, extended,

$$
D^{4} f_{0}=\sum \frac{\partial^{4} f_{0}}{\partial x_{r}{ }^{2} \partial x_{s}{ }^{2}} \quad \Im^{6} f_{0}=\sum \frac{\partial^{6} f_{0}}{\partial x_{r}{ }^{2} \partial x_{s}{ }^{2} \partial x_{t}{ }^{2}} \quad Q^{8} f_{0}=\sum \frac{\partial^{8} f_{0}}{\partial x_{r}{ }^{2} \partial x_{s}{ }^{2} \partial x_{t}{ }^{2} \partial x_{u}{ }^{2}}
$$

etc., the summations extending over all possible combinations of $r, s, t, \cdots$ with no two equal.

Labelling the symmetrical sets of points as in Part 2, we have likewise the expansions for sums of values of $f$ over the sets

Received December 30, 1959. This work was supported in part by the Office of Naval Research. 
(2.31) $\quad 0 \quad f_{0}$

(2.32)

$$
\begin{gathered}
\alpha(a) \quad 2 n f_{0}-\frac{4 h^{4} a^{4}}{4 !} D^{4} f_{0}+\frac{6 h^{6} a^{6}}{6 !} \Im^{6} f_{0}+\frac{4 h^{8} a^{8}}{8 !}\left(D^{8}-2 Q^{8}\right) f_{0} \\
-\frac{10 h^{10} a^{10}}{10 !}\left(D^{4} J^{6}-\mathcal{P}^{10}\right) f_{0} \\
-\frac{2 h^{12} a^{12}}{12 !}\left(2 D^{12}-3 J^{12}\right. \\
\left.-6 D^{4} Q^{8}+6 S^{12}\right) f_{0}+\cdots
\end{gathered}
$$

$$
\begin{aligned}
\beta(b) \quad 2 n(n & -1) f_{0}-\frac{8 h^{4} b^{4}}{4 !}(n-4) D^{4} f_{0}+\frac{12 h^{6} b^{6}}{6 !}(n-16) J^{6} f_{0} \\
& +\frac{8 h^{8} b^{8}}{8 !}\left\{(n+6) D^{8}-2(n-64) Q^{8}\right\} f_{0} \\
& -\frac{20 h^{10} b^{10}}{10 !}\left\{(n-4) D^{4} J^{6}-(n-256) P^{10}\right\} f_{0} \\
& -\frac{4 h^{12} b^{12}}{12 !}\left\{2(n-34) D^{12}-3(n+362) J^{12}\right. \\
& \left.\quad-6(n-728) D^{4} Q^{8}+6(n-1024) \mathfrak{S}^{12}\right\} f_{0}+\cdots
\end{aligned}
$$

(2.34) $\quad \gamma(c, d) \quad 4 n(n-1) f_{0}-\frac{8 h^{4}}{4 !}\left\{(n-1)\left(c^{4}+d^{4}\right)-6 c^{2} d^{2}\right\} D^{4} f_{0}$

$$
\begin{aligned}
& +\frac{12 h^{6}}{6 !}\left\{(n-1)\left(c^{6}+d^{6}\right)-15 c^{2} d^{2}\left(c^{2}+d^{2}\right)\right\} \Im^{6} f_{0} \\
& +\frac{8 h^{8}}{8 !}\left[\left\{(n-1)\left(c^{8}+d^{8}\right)-28 c^{2} d^{2}\left(c^{4}+d^{4}\right)+70 c^{4} d^{4}\right\} D^{8}\right. \\
& \left.\quad-2\left\{(n-1)\left(c^{8}+d^{8}\right)-28 c^{2} d^{2}\left(c^{4}+d^{4}\right)-70 c^{4} d^{4}\right\} Q^{8}\right] f_{0} \\
& +\ldots
\end{aligned}
$$

$$
\begin{aligned}
& \epsilon(e) \quad \frac{4}{3} n(n-1)(n-2) f_{0}-\frac{8 h^{4} e^{4}}{4 !}(n-2)(n-7) D^{4} f_{0} \\
& \quad+\frac{12 h^{6} e^{6}}{6 !}\left(n^{2}-33 n+122\right) J^{6} f_{0} \\
& + \\
& +\frac{8 h^{8} e^{8}}{8 !}\left\{(n-2)(n+13) D^{8}-2\left(n^{2}-129 n+1094\right) Q^{8}\right\} f_{0} \\
& +\cdots
\end{aligned}
$$

We recall that 0 is the origin, or centre of the square, $\alpha(a)$ includes all points with one coordinate $\pm a h$ and the rest zero, $\beta(b)$ has two coordinates each independently $\pm b h$ and the rest zero, $\gamma(c, d)$ has one coordinate $\pm c h$, another $\pm d h$ and the rest zero, and finally $\epsilon(e)$ has three coordinates each independently $\pm e h$ with the rest zero. 
3. Expansions over a Square. Such expansions are simpler since $\tau^{6} f_{0}, Q^{8} f_{0}$ etc., are absent. They can be obtained by analysis with the detached operators-in particular $D$; we proceed to obtain expansions with general terms.'

If $F(z)=u+i v$ is a function of a complex variable $z=x+i y$ then both $u$ and $v$ are harmonic functions satisfying $D_{x}^{2} \phi+D_{y}^{2} \phi=0$. Likewise, if $u$ is a harmonic function, it can be shown that $v$ exists such that $u+i v$ is a function of a complex variable. We then have

$$
D_{y} F=i F^{\prime}=i D_{x} F
$$

and

$$
D_{x} D_{y}=\mathscr{D}^{2}=i D_{x}^{2}=-i D_{y}^{2} .
$$

In order to develop expansions we therefore substitute

$$
D_{x}=i^{-1 / 2} \mathscr{D} \quad D_{y}=i^{1 / 2} \mathfrak{D} .
$$

Consider, firstly

$$
J=(2 h)^{-2} \int_{-h}^{h} \int_{-h}^{h} f(x, y) d x d y=\frac{1}{4 h^{2} D_{x} D_{y}}\left(e^{h D_{x}}-e^{-h D_{x}}\right)\left(e^{h D_{y}}-e^{-h D_{y}}\right) f_{0} .
$$

The operator is

$$
\left\{\begin{aligned}
\frac{\sinh h D_{x} \sinh h D_{y}}{h^{2} D_{x} D_{y}} & =\frac{\sinh i^{-1 / 2} h D \sinh i^{1 / 2} h D}{h^{2} D^{2}} \\
& =\frac{1}{2} \frac{\cosh \left(i^{1 / 2}+i^{-1 / 2}\right) h D-\cosh \left(i^{1 / 2}-i^{-1 / 2}\right) h D}{h^{2} D^{2}} \\
& =\frac{1}{2} \frac{\cosh \sqrt{2} h D-\cos \sqrt{2} h D}{h^{2} D^{2}}
\end{aligned}\right.
$$

whence

$$
J=\left(\frac{2}{2 !}+\frac{2^{3} h^{4}}{6 !} D^{4}+\frac{2^{5} h^{8}}{10 !} D^{8}+\cdots+\frac{2^{2 r+1} h^{4 r}}{(4 r+2) !} D^{4 r}+\cdots\right) f_{0} .
$$

Likewise

$$
\left\{\begin{aligned}
\sum_{\alpha(a)} f\left(x_{\alpha}, y_{\alpha}\right) & =\left(e^{a h D_{x}}+e^{-a h D_{x}}+e^{a h D_{y}}+e^{-a h D_{y}}\right) f_{0} \\
& =2\left(\cosh a h D_{x}+\cosh a h D_{y}\right) f_{0} \\
& =2\left(\cosh i^{-1 / 2} a h D+\cosh i^{1 / 2} a h D\right) f_{0} \\
& =4 \cosh \frac{a h}{\sqrt{2}} D \cos \frac{a h}{\sqrt{2}} D f_{0} \\
& =4\left[1-\frac{a^{4} h^{4}}{4 !} D^{4}+\frac{a^{8} h^{8}}{8 !} D^{8}+\cdots+(-1)^{r} \frac{a^{4 r} h^{4 r}}{(4 r) !} D^{4 r}+\cdots\right] f_{0}
\end{aligned}\right.
$$

and

$$
\left\{\begin{aligned}
\sum_{\beta(b)} f\left(x_{\beta}, y_{\beta}\right) & =4 \cosh b h D_{x} \cosh b h D_{y} f_{0} \\
& =4 \cosh i^{-1 / 2} b h D \cosh i^{1 / 2} b h D f_{0} \\
& =2(\cosh b h \sqrt{2} D+\cos b h \sqrt{2} D) f_{0} \\
& =4\left[1+\frac{2^{2} b^{s} h^{4}}{4 !} D^{4}+\frac{2^{\prime} b^{8} h^{8}}{8 !} D^{8}+\cdots+\frac{2^{2 r} b^{4 r} h^{4 r}}{(4 r) !} D^{4 r}+\cdots\right] f_{0}
\end{aligned}\right.
$$


We shall not use all the expansions given above in the present note, but it seems useful to set out the collected results for future use.

4. Lattice-point Formulas over a Square. We consider first formulas in two variables, and start with 9 points, putting $a=b=1$ and using the sets $0, \alpha(1)$, $\beta(1)$. We write

$$
J=I / 4 h^{2}=A_{0} f(0,0)+\sum A_{\alpha} f\left(x_{\alpha}, y_{\alpha}\right)+\sum A_{\beta} f\left(x_{\beta}, y_{\beta}\right)
$$

using $\left(x_{\alpha}, y_{\alpha}\right)$ etc. as typical sets of coordinates.

Using (3.5) to (3.7), we equate coefficients of $D^{4 r} f_{0}, r=0(1) 2$. This gives

$$
\left\{\begin{aligned}
A_{0}+4 A_{\alpha}+4 A_{\beta} & =1 \\
-4 A_{\alpha}+16 A_{\beta} & =\frac{4}{15} \\
+4 A_{\alpha}+64 A_{\beta} & =\frac{16}{45}
\end{aligned}\right.
$$

with correction term $\quad C=-\left(-4 A_{\alpha}+256 A_{\beta}-\frac{64}{91}\right) \frac{h^{12}}{12 !} D^{12} f_{0}$.

We obtain the formula

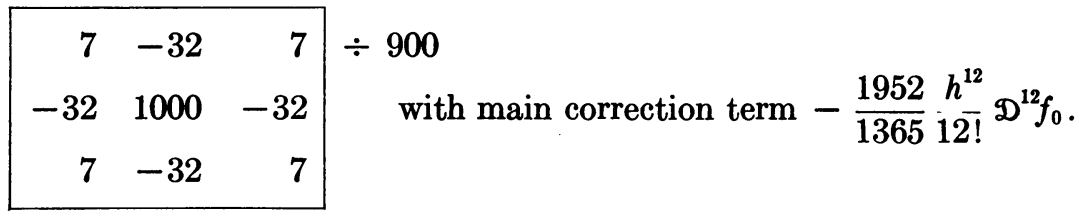

This formula is remarkably good. With the example of Part I, we have, writing $J^{\prime}=h^{2} J$

$J^{\prime}=\frac{1}{4} \int_{0}^{1.2} \int_{0}^{1.2} \sin x \sinh y d x d y=\frac{1}{4}(1-\cos 1.2)(\cosh 1.2-1)$

$$
\doteqdot 0.12922705907367511602
$$

Formula (4.3) gives

$$
J^{\prime} \doteqdot 0.12922705907283411029
$$

with $E=-0.0^{12} 84100573$ and $C=+0.0^{12} 84101633$.

5. Five-point Formulas. The high precision of (4.3) suggests that formulas of lesser precision, with fewer points, may be useful. We use the first two of (4.2) and take one of $A_{0}, A_{\alpha}, A_{\beta}$ to be zero.

(i) $A_{0}=0$ gives an eight-point formula with relatively poor precision.

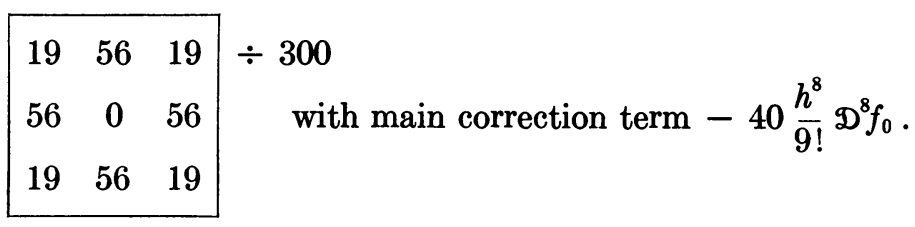

(ii) $A_{\alpha}=0$ gives

$$
\begin{array}{rrr}
1 & 0 & 1 \\
0 & 56 & 0 \\
1 & 0 & 1
\end{array} \quad \div 60 \quad \text { with main correction term }-\frac{32}{5} \frac{h^{8}}{9 !} D^{8} f_{0} .
$$


(iii) $A_{\beta}=0$ gives

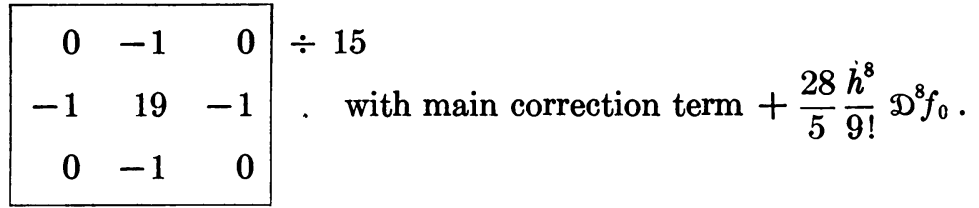

We observe that (5.2) and (5.3) combine in the proportions $\frac{7}{15}: \frac{8}{15}$ to give (4.3), though without an error estimate! Likewise $\frac{7}{3} \times(5.2)-\frac{4}{3} \times(5.3)$ gives (B) of Note I, and an estimate for the correction, namely $-\frac{112}{5} \frac{h^{8}}{9 !} D^{8} f_{0}$ when $f(x, y)$ is harmonic.

Another combination, that of (5.2) and (5.3) in equal proportions, gives a small correction term:

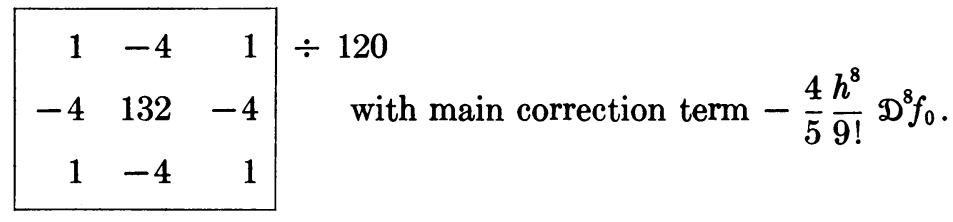

Again $4 \times(5.2)-3 \times(5.3)$ gives small multipliers:

$$
\begin{array}{rrr}
1 & 3 & 1 \\
3 & -1 & 3 \\
1 & 3 & 1
\end{array} \mid \div 15 \quad \text { with main correction term }-\frac{212}{5} \frac{h^{8}}{9 !} D^{8} f_{0} .
$$

Evidently (4.3) is most precise, but simultaneous use of (5.2) and (5.3) gives an idea of the precision attained, and readily yields the better result if desired. Formula (5.5) might be helpful with desk computing, but (5.1) has little to recommend it.

Numerical results for some of the formulas using the example of $\$ 4$ are as follows:

\begin{tabular}{c|c|r|r}
\hline Formula & Result $J^{\prime}$ & $10^{10} \times E$ & \multicolumn{1}{|c}{$10^{10} \times C$} \\
\hline$(5.1)$ & 0.1292272986 & +2395 & -2396 \\
$(5.2)$ & 0.1292270974 & +383 & -383 \\
$(5.3)$ & 0.1292270255 & -336 & +335 \\
$I(\mathrm{~B})$ & 0.1292271932 & +1341 & -1342 \\
$(5.4)$ & 0.1292270615 & +24 & -24 \\
\hline
\end{tabular}

6. General $n ; 2 n^{2}+1$ Points. We consider now the $n$-dimensional case, $n \geqq 3$, using lattice points $0, \alpha(1), \beta(1)$. In this case the term in $5^{6} f_{0}$ is relevant, and the $Q^{8} f_{0}$ term will appear in the error, except when $n=3$.

We equate coefficients of $f_{0}, D^{4} f_{0}, J^{6} f_{0}$ in the expansions resulting from use of (2.1), (2.31)-(2.33) in (4.1). We obtain 


$$
\left\{\begin{aligned}
A_{0}+2 n A_{\alpha}+2 n(n-1) & A_{\beta}=1 \\
-4 A_{\alpha}-8(n-4) & A_{\beta}=\frac{4}{15} \\
+6 A_{\alpha}+12(n-16) A_{\beta} & =\frac{16}{21}
\end{aligned}\right.
$$

while

$$
\begin{aligned}
C=-\left\{4 A_{\alpha}+8(n+6) A_{\beta}-\frac{16}{45}\right\} & \frac{h^{8}}{8 !} D^{8} f_{0} \\
& +\left\{8 A_{\alpha}+16(n-64) A_{\beta}+\frac{192}{45}\right\} \frac{h^{8}}{8 !} Q^{8} f_{0} .
\end{aligned}
$$

These yield

$$
A_{0}=\frac{-61 n^{2}+931 n+3780}{3780} \quad A_{\alpha}=\frac{61 n-496}{3780} \quad A_{\beta}=-\frac{61}{7560}
$$

with

$$
C=\frac{1198}{945} \frac{h^{8}}{8 !} D^{8} f_{0}+\frac{3619}{315} \frac{h^{8}}{8 !} Q^{8} f_{0}
$$

In particular

$$
\begin{array}{llll}
n=3 & A_{0}=\frac{12048}{7560} & A_{\alpha}=-\frac{626}{7560} & A_{\beta}=-\frac{61}{7560} \\
n=4 & A_{0}=\frac{13056}{7560} & A_{\alpha}=-\frac{504}{7560} & A_{\beta}=-\frac{61}{7560} \\
n=5 & A_{0}=\frac{13820}{7560} & A_{\alpha}=-\frac{382}{7560} & A_{\beta}=-\frac{61}{7560} \\
n=6 & A_{0}=\frac{14340}{7560} & A_{\alpha}=-\frac{260}{7560} & A_{\beta}=-\frac{61}{7560} .
\end{array}
$$

As a numerical illustration for $n=3$ we consider $J=\frac{1}{8} I=\frac{1}{8} \int_{-1}^{1} \int_{-1}^{1} \int_{-1}^{1} \cos \frac{3}{4} x \cos y \cosh \frac{5}{4} z d x d y d z$

$$
=\frac{16}{15} \sin \frac{3}{4} \sin 1 \sinh \frac{5}{4}=0.9800827 .
$$

Formula (6.33) gives $J \doteqdot 0.9799734$ with $E=-0.0000109$ and $C=+0.0000110$.

This result is less spectacular than that of $\$ 4$, for these reasons:

i) In $\$ 4, h=0.6$, here $h=1$, and the correction term in (4.3) contains a high power of $h$.

ii) The correction term in (6.2) is of order $h^{8}$, that in (4.3) is of order $h^{12}$

iii) The higher the number of dimensions, the more individual terms there are in $D^{8} f, D^{12} f$, etc. In (4.3) there is only one term in $D^{12} f$, in $(6.33)$ there are 9 in $D^{8} f$.

iv) The effect of larger interval $h$ is enhanced by the use of the factor $\frac{5}{4}$, which exceeds unity, in $\cosh \frac{5}{4} z$; this is only partially balanced by the factor $\cos \frac{3}{4} x$.

In spite of these points, the formula (6.2) seems a good one.

7. Quadrature over a Square; Specially Chosen Points. Since the expansions 
of $\S 3$ involve only cross-differences $D^{4} f_{0}$, it appears likely that use of sets of diagonal points $\beta$ will be more profitable than attempts to use sets $\alpha$. It turns out that sets $0, \alpha(a), \beta(b)$ and $0, \alpha(a)$ both fail to give real values of $\alpha$ if maximum precision is sought. On the other hand, we can get several formulas making use of any number of sets $\beta\left(b_{p}\right), p=0(1) r$, both with and without the point 0 .

We start first with $r$ sets $\beta\left(b_{p}\right)$, without the point 0 . We have to find the $2 r$ constants $A_{\beta_{p}}, b_{p}$ satisfying the equations

$$
\sum_{p=1}^{r} 4 A_{\beta_{p}} b_{p}^{4(s-1)}=\frac{1}{(2 s-1)(4 s-3)}=C_{s-1}, \quad s=1(1) 2 r
$$

obtained by substitution of (3.5) to (3.7) in

$$
J=\sum A_{\beta_{p}} f\left( \pm b_{p} h, \pm b_{p} h\right)
$$

and equating the coefficients of the first $2 r$ coefficients of $D^{4}$. Sundry powers of 4 have been cancellèd.

By familiar arguments, the $b_{p}{ }^{4}$ are roots of the equation

$$
\left|\begin{array}{ccccc}
1 & x & x^{2} & \cdots & x^{r} \\
C_{0} & C_{1} & C_{2} & \cdots & C_{r} \\
C_{1} & C_{2} & C_{3} & \cdots & C_{r+1} \\
\cdots & \ldots & \ldots & \ldots & \ldots \\
C_{r} & C_{r-1} & C_{r-2} & \cdots & C_{2 r-1}
\end{array}\right|=0 .
$$

These are the orthogonal polynomials for the weight function $w(x)=\frac{1}{2}\left(x^{-3 / 4}-x^{-1 / 2}\right)$ and range $0 \leqq x \leqq 1$. The first two are

$$
\left\{\begin{array}{l}
15 x-1=0 \\
819 x^{2}-438 x+11=0 .
\end{array}\right.
$$

The main correction term is obtained from the next power of $D^{4}$ and yields

$$
C=\left(C_{2 r}-\sum_{p=1}^{r} 4 A_{\beta_{p}} b_{p}^{8 r}\right) \frac{2^{4 r} h^{8 r} D^{8 r}}{(8 r) !} f_{0} .
$$

If the point 0 is included, our equations (7.1) are replaced by

$$
\left\{\begin{array}{l}
A_{0}+4 \sum_{p=1}^{r} A_{\beta_{p}}=1 \\
\sum_{p=1}^{r} 4 A_{\beta_{p}} b_{p}^{4 s}=\frac{1}{(2 s+1)(4 s+1)}=C_{s}, \quad s=1(1) 2 r
\end{array}\right.
$$

and the $b_{p}{ }^{4}$ are roots of the equation

$$
\left|\begin{array}{ccccc}
1 & x & x^{2} & \cdots & x^{r} \\
C_{1} & C_{2} & C_{3} & \cdots & C_{r+1} \\
C_{2} & C_{3} & C_{4} & \cdots & C_{r+2} \\
\cdots & \ldots & \ldots & \ldots & \ldots \\
C_{r+1} & C_{r+2} & C_{r+3} & \cdots & C_{2 r}
\end{array}\right|=0
$$


which are the orthogonal polynomials for the weight function $w(x)=\frac{1}{2}\left(x^{1 / 4}-x^{1 / 2}\right)$ for the range $0 \leqq x \leqq 1$. The first two are

$$
\left\{\begin{array}{l}
3 x-1=0 \\
17017 x^{2}-13650 x+1745=0 .
\end{array}\right.
$$

The main correction term is this time

$$
C=\left(C_{2 r+1}-\sum_{p=1}^{r} 4 A_{\beta_{p}} b_{p}^{8 r+4}\right) \frac{2^{4 r+2} h^{8 r+4}}{(8 r+4) !} D^{8 r+4} f_{0} .
$$

In each case the coefficients $A_{\beta_{r}}$ may be computed by standard methods.

8. Formulas for $r=1$. These have 4 and 5 points respectively

$$
\begin{array}{ccc}
A_{\beta}=\frac{1}{4} \quad b=15^{-1 / 4} & C=\frac{64}{225} \frac{h^{8} D^{8}}{8 !} f_{0} \\
A_{0}=\frac{4}{5} \quad A_{\beta}=\frac{1}{20} \quad b=3^{-1 / 4} & C=\frac{2816}{12285} \frac{h^{12} D^{12}}{12 !} f_{0} .
\end{array}
$$

Written out in full:

$$
\begin{aligned}
& J=\frac{1}{4} I=\frac{1}{4}\left\{f\left(15^{-1 / 4}, 15^{-1 / 4}\right)+\right. f\left(-15^{-1 / 4}, 15^{-1 / 4}\right) \\
&\left.+f\left(15^{-1 / 4},-15^{-1 / 4}\right)+f\left(-15^{-1 / 4},-15^{-1 / 4}\right)\right\} \\
& J=\frac{1}{4} I=\frac{4}{5} f(0,0)+\frac{1}{2 \sigma}\left\{f\left(3^{-1 / 4}, 3^{-1 / 4}\right)+f\left(-3^{-1 / 4}, 3^{-1 / 4}\right)\right. \\
&\left.\quad+f\left(3^{-1 / 4},-3^{-1 / 4}\right)+f\left(-3^{-1 / 4},-3^{1 / 4}\right)\right\}
\end{aligned}
$$

As a numerical test use

$J=\frac{1}{4} I=\frac{1}{4} \int_{-1}^{1} \int_{-1}^{1} \cos x \cosh y d x d y=\sin 1 \sinh 1=0.988897705762865$.

Formula (8.3) gives 0.9888906525 with

$$
E=-0.0000070533 \text { and } C=+0.0000070547
$$

and formula (8.4) gives 0.988897706241358 with

$$
E=+0.0^{9} 478493 \text { and } C=-0.0^{9} 478543 .
$$

9. Formulas for $r=2$. These have 8 and 9 points respectively

$$
\begin{array}{ll}
b_{1}=0.40316260305934689754 & A_{\beta_{1}}=0.22912306542816997222 \\
b_{2}=0.84439753192347874713 & A_{\beta_{2}}=0.02087693457183002778
\end{array}
$$

with main correction term $\frac{54592}{57014685} \times \frac{256}{16 !} h^{16} D^{16} f_{0}$

$$
\begin{aligned}
& b_{0}=0 \\
& A_{0}=0.69521808341292581989 \\
& b_{1}=0.63205020781879699524 \quad A_{\beta_{1}}=0.06686421854610538162 \\
& b_{2}=0.89531637912410697730 \quad A_{\beta_{2}}=0.00993126060066316340
\end{aligned}
$$


with main correction term $\frac{16832}{78975897} \times \frac{1024}{20 !} h^{20} D^{20} f_{0}$.

For

$$
J=\frac{1}{4} \int_{-1}^{1} \int_{-1}^{1} \cos x \cosh y d x d y
$$

formula (9.1) gives 0.98889770576285338396 with

$$
E=-0.0^{13} 1171243 \text { and } C=+0.0^{13} 1171555
$$

while formula (9.2) gives 0.98889770576286509647 with

$$
E=+0.0^{19} 9 \text { and } C=-0.0^{19} 90 .
$$

With formula (9.2) we find approximately 0.82447370907790316756 for

$$
\frac{1}{16} \int_{-2}^{2} \int_{-2}^{2} \cos x \cosh y d x d y=\sin 2 \sinh 2 \doteqdot 0.82447370907780915433
$$

with $E=+0.0^{13} 9401323$ and $C=-0.0^{13} 9406250$.

These formulae clearly have high precision, even with considerable values of $h$.

10. Quadrature over a Cube; Specially Chosen Points. The search for such formulas is more difficult in 3 or more dimensions. It seems that one or more extra available constants are needed in order to obtain real points. We shall not pursue this, but give one simple formula for three dimensions.

We find nothing convenient by use of points $\alpha(a)$, with or without 0 ; likewise 0 with $\beta(b)$ fails to give real points. We can, however, use 12 points $\beta(b)$ alone. We have then to satisfy

$$
\left\{\begin{array}{l}
2 n(n-1) A_{\beta}=1 \\
8 b^{4}(4-n) A_{\beta}=\frac{4}{15}, \quad \text { where } n=3 .
\end{array}\right.
$$

This yields $\quad b=(2 / 5)^{1 / 4}=0.795270728767051 \quad A_{\beta}=1 / 12$

with main correction term

$$
C=\left(156 b^{6} A_{\beta}+\frac{16}{21}\right) \frac{h^{6}}{6 !} \Im^{6} f_{0}=0.005626 h^{6} \jmath^{6} f_{0} .
$$

With the example of $\S 6$, with integrand $\cos \frac{3}{4} x \cos y \cosh \frac{5}{4} z(10.1)$ gives $J \doteqdot 0.97519$ with $E=-0.00489$ and $C=+0.00494$.

The only formula found that allows for the term $J^{6} f_{0}$ and has an error of order $h^{8}$ is (6.33), which needs 19 points. It is evident that further search is needed.

The University Mathematical Laboratory

Cambridge, England; and

The Digital Computer Laboratory

University of Illinois, Urbana, Illinois

1. J. C. P. Miller, "Numerical quadrature over a rectangular domain in two or more dimensions, Part I. Quadrature over a square, using up to sixteen equally spaced points," Math. Comp., v. 14, 1960, p. 13-20.

2. J. C. P. Miller, "Numerical quadrature over a rectangular domain in two or more dimensions, Part 2. Quadrature in several dimensions, using special points," Math. Comp., v. 14,1960 , p. $130-138$. 